сигналов. При всей своей повсеместной распространённости данная система не идеально и у неё есть свои достоинства и недостатки.

Достоинства:

— ЭМГ является хорошо изученным и проверенным методом диагностики;

— не требует предварительной подготовки;

- может проводиться неоднократно;

- отсутствие сильных болей во время и после процедуры (процедура сопровождается легким покалыванием).

Недостатки:

— достаточно длительная процедура (от получаса до часа);

- диагностика достаточно информативна только в комплексе с иными обследованиями;

- при использовании игольчатых электродов возможны неприятные ощущения;

- противопоказано людям с нервными расстройствами и проблемами со свертываемостью крови (при игольчатой ЭМГ).

Данная система является знаковой для изучения периферического нейромоторного аппарата и медицины в целом.

$$
* * *
$$

1. Гехт Б.М. Теоретичекая и клиническая электромиография. -Ленинград:Наука, 1990.-230с.

2. Модель эмг. Изучение принципов работы электромиографа.-Тула: ТУЛАНАУЧПРИБОР,2012.-59c.

3. Эрхан А. Разработка и контроль лечебного робота-тренажера для реабилитации нижних конечностей. Турция:Мехатроника и инженирия,2011.-509c.

\title{
Смоленцева T.E.
}

Анализ поведения параметров, влияющих на функционирование сложных систем ФГБОУ ВО «Липеикий государственный технический университет» (Россия, Липеик)

doi: $10.18411 / \mathrm{sr}-10-12-2018-13$

idsp: sciencerussia-10-12-2018-13

\section{Аннотация}

В работе рассмотрены параметры оказывающие влияние на поведение сложных организационных систем. Выявлены факторы, без учета которых невозможно корректно проводить оптимизацию структур организационных сложных систем. На основе выводов полученных при анализе рассмотренных вопросов проведена оценка процесса функционирования иерархических сложных систем.

Ключевые слова: уровень интенсивности функционирования, напряженность функционирования, иерархические многоуровневые организационные системы.

Для анализа функционирования и формирования структурных элементов рассматриваемых сложных систем необходимо:

— определить вид системы (организационные, экономические, технические);

- степень иерархичности системы;

- уровень масштабности задач;

- определить множество процедур, на основе которых осуществляется решение задач;

- время, требуемое на решение задач;

— объект воздействия (внешний, внутренний);

— на основе чего осуществляется формализация; 
- - вид информации.

Определение целей является основой для эффективного функционирования систем. В свою очередь цели определяют стратегию и тактику управления этой системой, формируют структуру, которая предполагает разработку СУ-системы управления и ОУ объект управления.

Семиотический подход, можно применять, когда о среде управления имеются частичные данные, а устанавливается взаимосвязь между множеством параметров и средой управления. В динамике формируются множества $\{\mathrm{v}\}$ (где - множество способов разбиения на классы состояний ИМОС $\mathrm{V}=\{\mathrm{v} 1, \mathrm{v} 2, \ldots, \mathrm{vk}\})$ и $\{\mathrm{M}\}$ (где $\{\mathrm{M}\}-$ множество математических моделей поиска управленческих решений при множестве математических моделей поиска решений).

Процесс разработки ИСС будет более объективным, если имеются статистические данные о ее функционировании, если таких данных нет, то привлекаем экспертов. Классическое решение проблемы: теория принятия решения, теория игр.

Оценку процесса функционирования иерархических сложных систем (ИСС) необходимо осуществлять по нормативным параметрам $x^{H}(t)$ в момент времени $t$. Некоторые цели, определенные на основе нормативных документов, являются базовыми и актуальными параметрами $x^{d}{ }_{i}(t), j \in v^{d}$ по которым и производится анализ функционирования ИСС $[1,2]$. На микроуровне функционирование элементов рассматриваемых систем с их нормативными параметрами могут корректироваться по их интенсивности функционирования.

Для определения необходимой меры пространства нормативов $x^{H}(t)$ требуется отслеживать динамику изменения этих нормативов, в момент планирования $t-\Delta t$ за счет скорости изменения параметра $s_{i j}(t-\Delta t), i=1, n ; j=1, m$ у каждого элемента $s_{i j}(t-\Delta t)$ и предположим, что скорость изменения параметра $j$ в течение $\Delta t$ не меняется.

Уровень интенсивности функционирования (УИФ) для достижения норматива $x^{H}{ }_{i j}$ описывается выражением

$$
\Phi_{i j}^{H}(t)=\left(x_{i j}^{H}(t)-x_{i j}(t-\Delta t)\right) / s_{i j}(t-\Delta t)
$$

Считаем, что $\Phi_{i j \text {, }}^{H_{1}}$ есть напряженность функционирования (НФ) по соответствующим параметрам. УИФ значения $i$-го элемента по $j$-му параметру определяется выражением:

$$
\Phi_{i j}(t)=\left(x_{i j}(t)-x_{i j}(t-\Delta t)\right) / s_{i j}(t-\Delta t) .
$$

Процесс функционирования будет устойчивым, тогда когда значения параметров этой системы будут иметь предельные значения, а также необходимо определить максимальную функцию $\Phi_{i}^{*}(t)$, которая не должна превышать значения УИФ $\Sigma \Phi_{i j}^{H}(t)$ и вычислять нормативы на основе выражения[3]:

$$
\sum_{j=1}^{m} \Phi_{i j}(t)=\Phi_{i}^{0}(t), \quad j=1, m ; i=1, n .
$$

где $\Phi_{i}^{0}(t)$ есть оценки $\Phi_{i}^{*}(t)$ определяемые экспертным путем на основе уровня напряженности функционирования соответствующих элементов иерархических систем в момент планирования $t-\Delta t .[2,4]$

Значения параметров $x^{H}(t)$ необходимо ограничить или на основе экспертных оценок или значений каждого параметра $x_{i j}$, любой из которых имеет максимальное значение $x_{i j}^{\max }(t)$ на рассматриваемом временном интервале.

Для базовых нормативов существуют ограничения

$$
\sum_{i=1}^{m} x_{i j}^{H}(t)=x_{j}^{d}, \quad j \in V^{d} \text {. }
$$


В этом случае значения нормативов $x^{H}(t)$ при сформированных ограничениях определяются, максимизацией критерия эффективности организационных систем, который оценивается суммарным уровнем НФ:

$$
\Phi_{j}=\sum_{i=1}^{n} \sum_{j=1}^{m} \Phi_{i j}^{H}(t) .
$$

На основе полученных результатов пришли к выводу о том, что функционирование организационных иерархических сложных систем будет устойчивым, в случае, когда все рассматриваемые параметры будут принимать предельные значения.

1. Замятина, О.М. Моделирование систем / О.М. Замятина // Издательство ТПУ.-2009.-c. 204.

2. Жиляков Е.Г. Компьютерная кластеризация совокупности аддитивных математических моделей взаимосвязанных процессов / Е.Г. Жиляков, В.А. Ломазов, В.И. Ломазова // Вопросы радиоэлектроники. Сер. ЭВТ. - 2011. - Вып.1. - С. 115-119.

3. T E Smolentseva, Mathematical Models to Determine Stable Behavior of Complex Systems / V I Sumin, A V Dushkin, T E Smolentseva// International Conference Information Technologies in Business and Industry 2018IOP Conf. Series: Journal of Physics: Conf. Series 1015 (2018) 032136.

4. Смоленцева Т.Е. Формирование структуры иерархических многоуровневых организационных систем// Сумин В. И., Смоленцева Т.Е./ Информационные технологии.- 2017, Москва. Издательство «Информационные технологии», том №23, №6 стр. 476-480.

\section{Смоленцева T.E. \\ Применение иерархических структурных моделей для оптимизации распределения задач}

ФГБОУ ВО «Липеиякий государственный технический университет»

(Россия, Липецк)

doi: $10.18411 / s r-10-12-2018-14$

idsp: sciencerussia-10-12-2018-14

\section{Аннотация}

В работе рассмотрен процесс формирования сложных структурных моделей. Проанализирована эффективность применения А-схем при формировании моделей. Рассмотрен вопрос распределения функциональных задач в иерархических сложных системах. На основе выводов полученных при анализе поставленных вопросов проведена оценка равновесного распределения функциональных задач между сотрудниками рассмотренной системы.

Ключевые слова: равновесное распределение, функциональные задачи, многоуровневые структурные модели.

Существующие системы моделирования обладают очень фиктивного возможностями в процессе математических происходит решения, однако имеют ряд существенных ограничений, не параметрами эффективно может весь спектр стоящих перед ними задач. В связи с этим всё большую значимость приобретает решение задачи по разработке принципов и виде построения систем таким сложных технических объектов, удовлетворяющих требованиям к выдаваемый инструментарию пяти.

Применение обобщенной математической схемы моделирования, т.е. А - схемы объекта являться тем времени, на котором базируется таким многоуровневых моделей. Стандартная описания представления исследуемого процесса в виде А-схемы приводит к унификации не только алгоритмов имитации, но и к возможности применять стандартные методы обработки и анализа результатов моделирования системы $\mathrm{S}$. 